\title{
Interaction Techniques for Hybrid Piles of Documents on Interactive Tabletops
}

\author{
Mohammadreza Khalilbeigi \\ Telecooperation Group \\ Darmstadt University of Technology \\ khalilbeigi@tk.informatik.tu- \\ darmstadt.de \\ Jürgen Steimle \\ Telecooperation Group \\ Darmstadt University of Technology \\ steimle@tk.informatik.tu- \\ darmstadt.de \\ Max Mühlhäuser \\ Telecooperation Group \\ Darmstadt University of Technology \\ max@tk.informatik.tu- \\ darmstadt.de
}

\begin{abstract}
Piling is a highly common activity for the casual organization of documents. Today's tabletops do not offer sufficient support for piling, particularly in hybrid settings where both digital documents and paper documents are used on the same surface. We contribute several techniques for interacting with hybrid piles of printed and digital documents on tabletops. By employing a soap bubble metaphor and by using paper as a tangible control for the hybrid pile, these allow easy creating and rearranging piles while maintaining the flexibility of traditional paper piles.
\end{abstract}

\section{Keywords}

Document, pile, interactive surface, paper, tabletop.

\section{ACM Classification Keywords}

H5.2. User Interfaces.

\section{General Terms}

Design.

\section{Introduction}

Piling is one of the most prominent forms of organizing documents in our everyday life [4]. This physical practice has been imitated in digital grouping concepts by 
offering various sophisticated gesture-based interaction techniques for piles of digital documents.

However, many knowledge work practices consist of a combined use of both digital and physical documents (which we call hybrid use). In this paper, we present hybrid piling techniques which support piling of printed and digital documents in any combination on interactive tabletops.

In an exploratory user study [11], we found that the main representational form for organizing printed and digital documents on tabletops is the hybrid pile. This is a layered arrangement of (partially or entirely) overlapping digital and physical documents. While all participants intuitively and easily created hybrid piles, it became evident that more explicit support of hybrid piles is needed: The user should be able to interact with a pile as with one single entity, e.g. for moving, while preserving the flexibility of individual documents. Moreover, users need to quickly obtain awareness of the digital documents of a hybrid pile, as this has been intuitively performed with physical papers.

Based on the design insights from the user study, we present a soap bubble metaphor to permit flexible reorganization and navigation of hybrid piles as with physical documents. We also contribute a set of novel hybrid interaction techniques to enable fluid transitions between different representational modes. Finally, we discuss the emerging advantages and challenges of treating traditional paper as tangible objects to control virtual documents in different piling activities.

\section{Related Work}

Early studies $[2,3]$ investigated and identified the advantages of piling versus filing on office worker desktops. It was found that piling is a lightweight, casual activity, involving less overhead than filing. In order to make the use of computers more natural, it was suggested to offer more support for piling in today's desktop computer systems. Mander et. al. [4] developed a prototype which explored the piling metaphor by proposing a set of gestures and interaction techniques for browsing and manipulating piles.

Inspired by [4], Agarawala et. al. [5] proposed a more comprehensive prototype which aimed to bring more realism to the today's rigid virtual desktops by adding physics simulation and using piling instead of filing as the fundamental organization structure. In this way, virtual objects can be dragged and tossed around in a realistic fashion. Moreover, a set of pen-based interaction and visualization techniques enable users to create and manipulate piles of virtual objects. In [10] an interface for grouping graphical objects is proposed. The approach relies on spatial clustering and visualizes clusters with a rotund bubble surrounding the objects.

There exist number of systems, which consider the issue of digital grouping and piling on interactive tabletops. Storage Bins [6] facilitate the casual and mobile storage of objects on interactive tabletops. Their mobility and adjustability in size and shape permit users to share resources and transitions between resources on tabletops. In order to support navigation, repositioning and reorganization of digital piles on tabletops, [7] proposed three stylus-based interaction techniques. These techniques are based on transparency [8] and expand- 


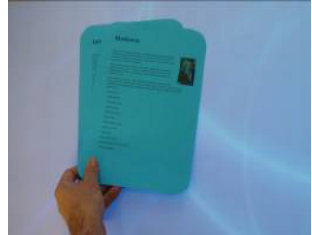

(a)

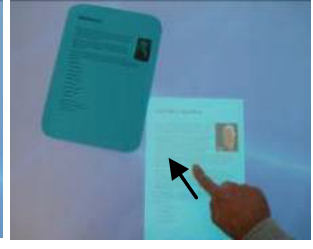

(b)

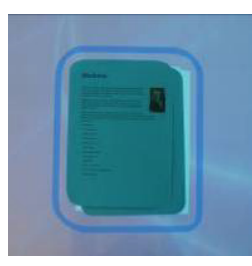

(c)
Figure 1. Pile creation by placing physical on digital documents (a) or dragging digital under physical one (b). (c) The Hybrid pile formed.

ing [9] ideas to effectively reveal the elements hidden in a stack of digital elements.

\section{Supporting Hybrid Piles}

We leverage a soap bubble metaphor, inspired from bubble clusters [10]. The soap bubble metaphor is a group visualization technique based on the spatial proximity of documents to each other. Once a hybrid group formed, an elastic bubble is displayed on the tabletop that surrounds the contour of the group (Fig. 1). This provides clear feedback about which documents belong to the group. Moreover, the flexible form of the bubble provides for flexible rearrangements of the documents contained within the group. Two bubbles join and become one large bubble if they get in touch; in the reverse way, one bubble can also be divided into two bubbles. Leveraging this flexible nature, we use soap bubbles to continuously provide unambiguous feedback about the hybrid grouping of documents and to allow easily and flexibly creating and rearranging groups.

In contrast to pure digital bubble clusters designed for interacting with a mouse [10], our concept addresses hybrid interaction with both physical and digital items. This allows more flexible ways of interaction, such as
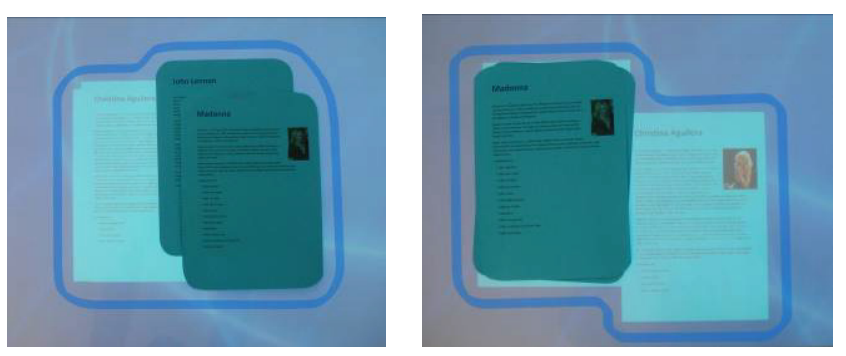

Figure 2. Flexible reorganization of the elements of a hybrid pile. The soap bubble adapts itself to the arrangements of the pile

using physical items as a graspable handle for the hybrid group (see below).

The soap bubble metaphor facilitates the following piling activities in the hybrid setting:

- Creating a group: By placing (or dragging) documents onto each other (see Fig. 1), a hybrid group is formed or a new item is added to an existing hybrid group. A bubble is automatically displayed around all documents containing to the same group. In addition we are also investigating more stable forms of grouping. By attaching several sheets of paper with a physical paper clip, a stable physical grouping is created, to which digital documents can be added as described above.

- Flexible reorganization: The soap bubble visualization allows very flexible arrangements of the documents contained in a group. Individual documents within a group can be displaced (see Fig. 2, for instance placed one besides the other for reading and comparison). The bubble flexibly adapts its shape to the new contours of the group. In contrast to [10], it is not only possible to interact with one single item at a 

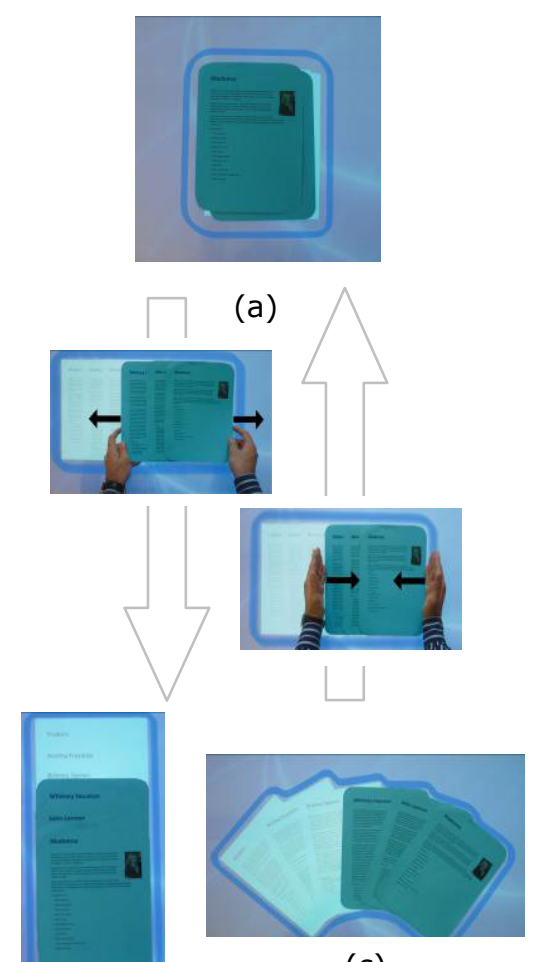

(c)

(b)

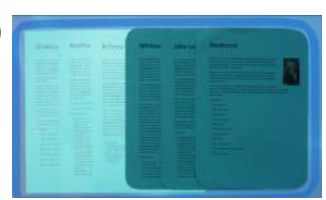

(d)

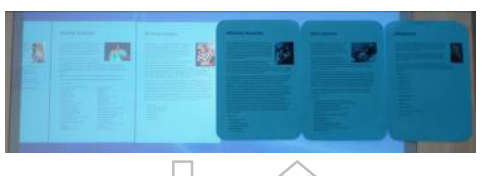

(e) 1

Figure 3. Fluid transitions for hybrid piles, from the compact form (a) to intermediate forms (top-to-down (b), fan out (c) and left-to-right (d)), to a full juxtaposition (e)
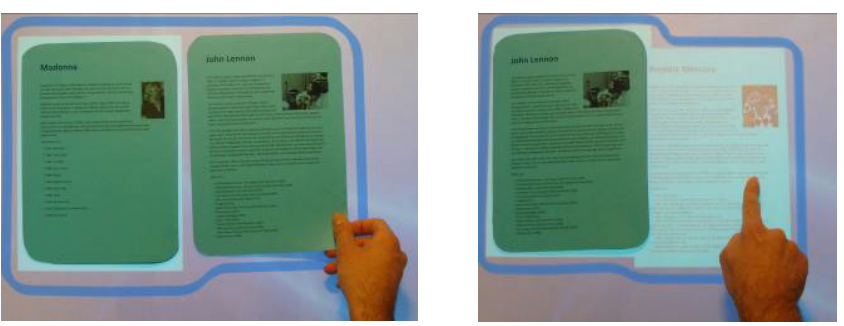

Figure 5. Moving individual physical or digital documents, without affecting the entire pile

time. With bimanual and tangible interactions, the user can grasp and move several items very easily.

- Removing an item: If a document of a group is relocated to be far from the other documents of the group, the single bubble of the group gradually splits into two separate bubbles (see Fig. 4). While dragging the document away, the user gets continuous feedback to indicate when the document gets separated from the group because the connecting part of the bubble will grow smaller. If the distance exceeds a threshold, the document(s) is/are removed from the group and form(s) a separate document or group.

\section{Fluid Representational Transitions}

We observed two main representational forms of groups:

1) a "tidy" pile of documents, which affords interaction with the group as whole, e.g. moving, and

2) a juxtaposition or partially overlapping arrangement, which affords getting an overview on the documents, reading and comparing them.

Groups of paper documents allow fluid transitions between both these representations. We frequently ob-
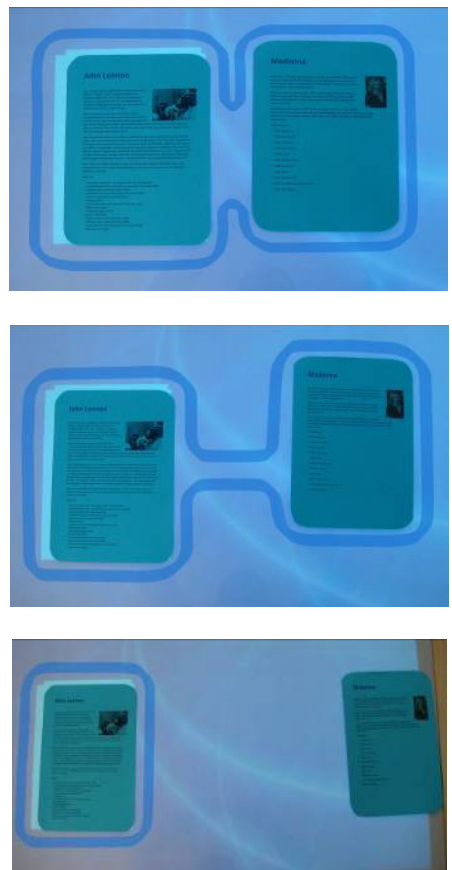

Figure 4. Removing a document from a hybrid pile. The soap bubble continuously visualizes the state of the pile.

served participants performing these transitions with one quick and intuitive movement. In contrast, in the digital and in the hybrid case, these transitions are much harder to perform, as each digital document has to be individually moved. In these cases, no transitions were made by the participants.

Our design leverages the many degrees of freedom of the tangible control to offer fluid transitions between hybrid group representations. In order to arrange all documents of a hybrid group in a particular representation, the user has to arrange only the physical part in this manner. The digital documents of the group are automatically relocated and rotated to form the same 
representation. Hence, these hybrid transitions are as easy to perform as with pure paper. They are depicted in Fig. 3.

These transitions can be used for getting an overview on all documents of a hybrid pile. Moreover, they allow quickly switching between different forms of interaction (e.g. moving a group vs. comparing the documents).

\section{Moving Hybrid Piles}

The ability to move and drag documents on the tabletop plays an important role in optimally managing the workspace. In our study we observed that physical piles were relocated very frequently by moving or pickingand-dropping. In contrast, digital and hybrid groups were never moved because suitable functionality was missing.

We want to leverage paper as a tangible control for easy moving of hybrid groups. However, this concept raises a conceptual challenge. If the user moves documents, it is not clear if she wants to interact with these documents using them as first-class objects (for example for reorganizing the pile as described above) or if she wants to use them as a tangible handle for the entire pile. Our design resolves this ambiguity as follows:

- If individual documents, but not the entire physical pile, are moved, the documents are treated as firstclass objects, i.e. they are rearranged without affecting the remainder of the pile. (represented in Fig. 5)

- If all documents of one entire physical pile are moved or picked-and-dropped (see Fig. 6) together, they are considered as a tangible handle of the entire hybrid pile. Automatically the digital part of the hybrid
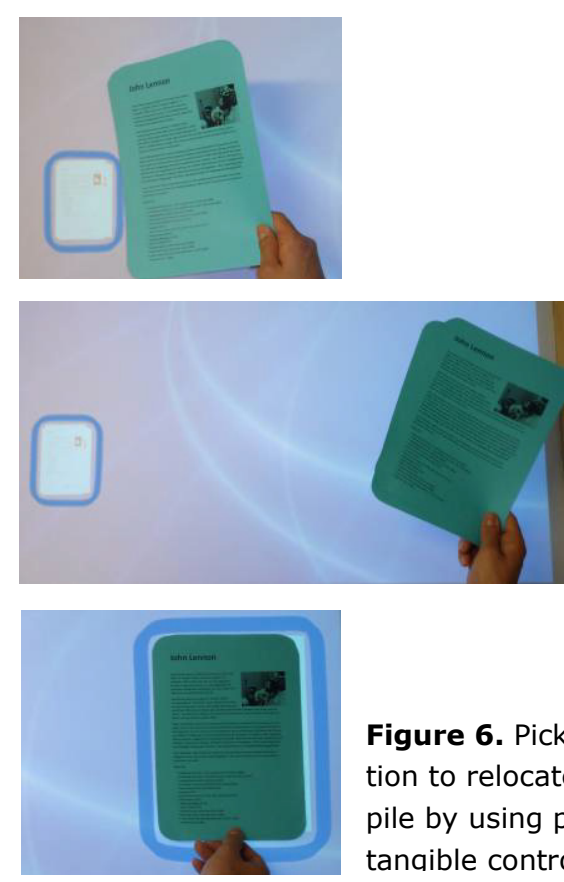

Figure 6. Pick-and-drop interaction to relocate an entire hybrid pile by using paper documents as tanqible controls.

pile is relocated according to the movement of the physical part. In the special case that the user wants to move all documents of the physical part at the same time, but without affecting the digital part, she can hold one of the parts at a fix position while moving the other part away. Then both parts can be moved in separation. They can be easily reconnected by simply placing them onto each other as of creation.

A second challenge with moving documents in the hybrid setting is generated by the presence of physical paper on the display. Digital items cannot be dragged over these occluded positions. To move documents across physically occluded areas, we propose a flipunder gesture. This is a bi-manual gesture, one finger 
specifying the destination and the other finger flipping the digital objects in that direction. This gesture also enables the users to quickly create a group of digital documents by specifying the center of group and dragging multiple digital documents to the group.

\section{Summary and Future Work}

In this paper we presented intuitive and lightweight visualization and interaction techniques to facilitate the manipulation of hybrid piles. In contrast to previous systems, our techniques do not require any specific gestures to manipulate piles of documents nor do they restrict the interaction with groups of documents to a specific area of the tabletop. Inspired by nature, we introduced a soap bubble visualization concept that flexibly leverages the spatial proximity of the elements of a pile. In this way, users can intuitively create hybrid piles by placing or dragging documents over each other and have high flexibility in reorganizing piles. Using paper documents as tangible controls for hybrid groups provides for easy and fluid transitions between different pile modes as well as for easy moving of hybrid piles.

We are currently implementing a tabletop system which coherently integrates these interaction and visualization techniques. We follow a marker tracking approach to keep track of the locations of physical documents. Our system uses above and beneath cameras to detect fiducial markers. For the near future, we plan to conduct a comprehensive user study.

\section{References}

[1] Wellner, P. Interacting with Paper on the DigitalDesk. Comm. ACM, 36(7), 1993, 87-96.

[2] Malone, T. How do people organize their desks?: Implications for the design of office information sys- tems. ACM Trans. on Info. Systems, 1(1), 1983, 99112.

[3] Whittaker, S. \& Hirschberg, J. The character, value, and management of personal paper archives. ACM Trans. on CHI, 8(2), 2001, 150-170.

[4] Mander, R., Salomon, G., and Wong, Y. Y.: A "Pile" Metaphor for Supporting Casual Organization of Information. Proc. CHI '92, 627-634.

[5] Agarawala, A. \& Balakrishnan, R. Keepin' it Real: Pushing the Desktop Metaphor with Physics, Piles and the Pen. Proc. CHI'06, 1283-1292.

[6] Scott, S.D., Carpendale, S., \& Habelski, S. Storage Bins: Mobile Storage for Collaborative Tabletop Displays. IEEE Computer Graphics \& Applications, 2005, 58-65.

[7] Aliakseyeu, D.; Subramanian, S.; Lucero, A. \& Gutwin, C. Interacting with piles of artifacts on digital tables. Proc. of the working conference on advanced visual interfaces, 2006.

[8] Bier, E., Maureen, S., Ken, P., Buxton, W., DeRose, T. Toolglass and magic lenses: the see-through interface. Proc. SIGGRAPH '93. ACM Press, 1993, 73-80. [9] Keller, I. For inspiration only. PhD thesis, Delft University of Technology, The Netherlands, 2005.

[10] Watanabe, N. Washida, M. \& Igarashi, T. Bubble clusters: an interface for manipulating spatial aggregation of graphical objects. Proc. UIST '07, 173-182.

[11] Steimle, J., Khalilbeigi, M. \& Mühlhäuser, M. Hybrid groups of printed and digital documents on tabletops: A Study. Proc of CHI'2010 Extended Abstract. 